\title{
Solar-System-Wide Significance of Mars Polar Science
}

A White Paper submitted to the Planetary Sciences Decadal Survey 2023-2032

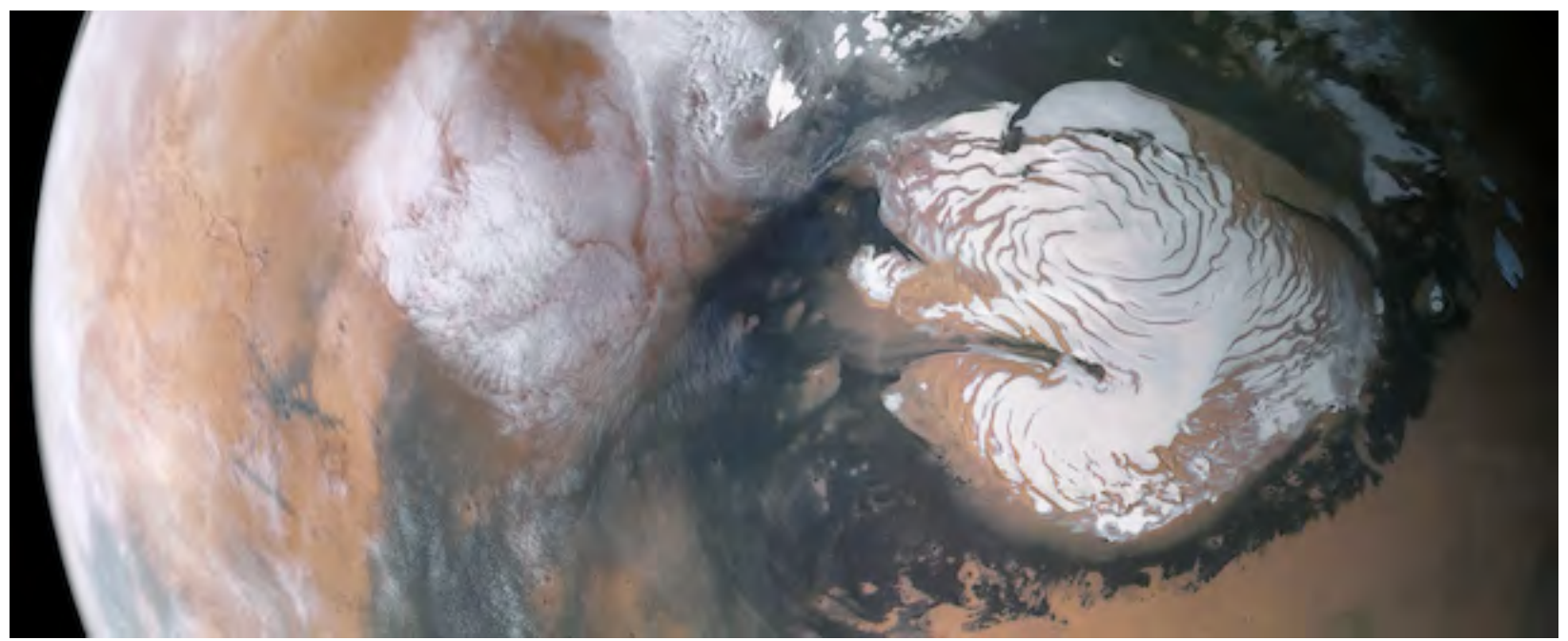

\section{Point of Contact:}

Isaac B. Smith (ibsmith@yorku.ca)

Phone: 647-233-3374

York University and Planetary Science Institute

4700 Keele St, Toronto, Ontario, Canada

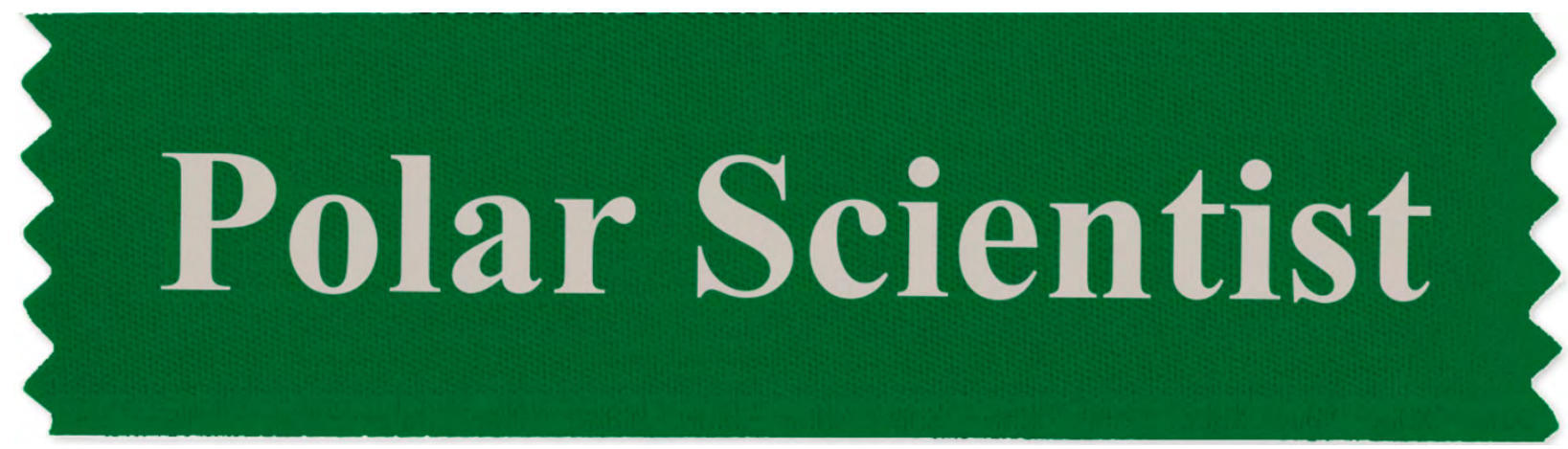

Acknowledgements: A portion of the research was carried out at the Jet Propulsion Laboratory, California Institute of Technology, under a contract with the National Aeronautics and Space Administration (80NM0018D0004). 
This list includes many of the hundreds of current students and scientists who have made significant contributions to Mars Polar Science in the past decade. Every name listed represents a person who asked to join the white paper or agreed to be listed and provided some comments.

\section{Author List:}

\begin{tabular}{|c|c|c|c|}
\hline I. B. Smith & York University, PSI & W. M. Calvin & University of Nevada Reno \\
\hline D. E. Smith & Massachusetts Institute of Technology & C. Hansen & Planetary Science Institute \\
\hline S. Diniega & Jet Propulsion Laboratory, Caltech & A. McEwen & Lunar and Planetary Laboratory \\
\hline N. Thomas & Universität Bern & D. Banfield & Cornell University \\
\hline T. N. Titus & U.S. Geological Survey & P. Becerra & Universität Bern \\
\hline M. Kahre & NASA Ames Research Center & F. Forget & Sorbonne Université \\
\hline M. Hecht & MIT Haystack Observatory & S. Byrne & University of Arizona \\
\hline C. S. Hvidberg & University of Copenhagen & P. O. Hayne & University of Colorado LASP \\
\hline J. W. Head III & Brown University & M. Mellon & Cornell University \\
\hline B. Horgan & Purdue University & J. Mustard & Brown University \\
\hline J. W. Holt & Lunar and Planetary Laboratory & A. Howard & Planetary Science Institute \\
\hline D. McCleese & Caltech & C. Stoker & NASA Ames Research Center \\
\hline P. James & Space Science Institute & N. E. Putzig & Planetary Science Institute \\
\hline J. Whitten & Tulane University & P. Buhler & Jet Propulsion Laboratory \\
\hline A. Spiga & Sorbonne Université & M. Crismani & University of Colorado \\
\hline K. M. Aye & University of Colorado LASP & A. Portyankina & University of Colorado LASP \\
\hline R. Orosei & Istituto di Radioastronomia & A. Bramson & Purdue University \\
\hline J. Hanley & Lowell Observatory & M. Sori & Purdue University \\
\hline O. Aharonson & Weizmann Institute of Science, PSI & S. Clifford & Planetary Science Institute \\
\hline H. Sizemore & Planetary Science Institute & G. Morgan & Planetary Science Institute \\
\hline B. Hartmann & Planetary Science Institute & N. Schorghofer & Planetary Science Institute \\
\hline R. Clark & Planetary Science Institute & D. Berman & Planetary Science Institute \\
\hline D. Crown & Planetary Science Institute & F. Chuang & Planetary Science Institute \\
\hline M. Siegler & cience Institute & E. N. Dobrea & ry Science Ins \\
\hline K. Lynch & Lunar and Planetary Institute/USRA & R. W. Obbard & SETI Institute \\
\hline M. R. Elmaary & University of London, Birkbeck & D. Fisher & Simpatico Canada \\
\hline A. Kleinboehl & Jet Propulsion Laboratory, Caltech & M. Balme & Open University \\
\hline B. Schmitt & University Grenoble & M. Daly & York University \\
\hline R. C. Ewing & Texas A\&M University & \multirow{2}{*}{\multicolumn{2}{|c|}{$\begin{array}{l}\text { K. E. Herkenhoff U.S. Geolo } \\
\text { S. D. Guzewich NASA GSFC }\end{array}$}} \\
\hline L. Fenton & SETI Institute & & \\
\hline M. Koutnik & University of Washington & J. Levy & Colgate University \\
\hline R. Massey & Durham University & A. Łosiak & Stanisław Leszczycki Institute \\
\hline V. Eke & Durham University & D. Goldsby & University of Pennsylvania \\
\hline A. Cross & Woods Hole Institute & T. Hager & University of Pennsylvania \\
\hline S. Piqueux & Jet Propulsion Laboratory & A. Kereszturi & Hungarian Academy of Sciences \\
\hline K. Seelos & JHU Applied Physics Laboratory & S. Wood & Planetary Science Institute \\
\hline E. Hauber & German Aerospace Center (DLR) & C. Amos & Planetary Science Institute \\
\hline P. Russell & University of California Los Angeles & R. Jaumann & Freie Universität Berlin \\
\hline G. Michael & Freie Universität Berlin & S. Conway & Université de Nantes \\
\hline A. Khayat & Univ of Maryland College Park & S. Lewis & The Open University UK \\
\hline G. Luizzi & NASA GSFC & G. Martinez & Lunar and Planetary Institute \\
\hline K. Mesick & Los Alamos National Laboratory & L. Montabone & Space Science Institute \\
\hline A. Johnsson & University of Gothenburg & A. Pankine & Space Science Institute \\
\hline C. Phillips-Land & der Southwest Research Institute & P. Read & University of Oxford \\
\hline
\end{tabular}




\begin{tabular}{|c|c|c|c|}
\hline L. Edgar & U.S. Geological Survey & K. Zacny & Honeybee Robotics \\
\hline A. McAdam & NASA GSFC & A. Rutledge & Northern Arizona University \\
\hline T. Bertrand & NASA Ames Research Center & J. Widmer & Representing Self \\
\hline D. Stillman & Southwest Research Institute & A. Soto & Southwest Research Institute \\
\hline Z. Yoldi & University of Copenhagen & R. Young & UAE University \\
\hline A. Svensson & University of Copenhagen & L. Sam & University of Aberdeen \\
\hline M. Landis & University of Colorado, Boulder & A. Bhardwaj & University of Aberdeen \\
\hline M. Chojnacki & Planetary Science Institute & E. Kite & University of Chicago \\
\hline P. Thomas & Cornell University & J. Plaut & Jet Propulsion Laboratory \\
\hline J. Bapst & Jet Propulsion Laboratory & S. Milkovich & Jet Propulsion Laboratory \\
\hline J. Whiteway & York University & J. Moores & York University \\
\hline C. Rezza & York University & R. Karimova & York University \\
\hline I. Mishev & York University & A. Van Brenen & York University \\
\hline P. Acharya & York University & J. Chesal & York University \\
\hline A. Pascuzzo & Brown University & E. Vos & Weizmann Institute of Scienc \\
\hline G. Osinski & University of Western Ontario & C. Andres & University of Western Ontario \\
\hline C. Neisch & University of Western Ontario, PSI & S. Hibbard & University of Western Ontario \\
\hline P. Sinha & Purdue University & J. P. Knightly & University of Arkansas \\
\hline S. Cartwright & University of Reno, Nevada & S. Kounaves & Tufts University \\
\hline C. Orgel & ESA ESTEC & M. Skidmore & Montana State University \\
\hline J. MacGregor & NASA GSFC & R. Staehle & Jet Propulsion Laboratory \\
\hline J. Rabassa & CADIC-CONICET & C. Gallagher & University College Dublin \\
\hline A. Coronato & CADIC-CONICET & A. G. Galofre & Arizona State University \\
\hline J. Wilson & Applied Physics Laboratory & L. McKeown & Natural History Museum UK \\
\hline N. Oliveira & Valongo Observatory & P. Fawdon & Open University \\
\hline U. Gayathri & SETI Institute & C. Stuurman & Jet Propulsion Laboratory \\
\hline C. Herny & Universität Bern & F. Butcher & University of Sheffield \\
\hline F. Bernardini & Planetary Science Institute Affiliate & M. Perry & Planetary Science Institute \\
\hline R. Hu & Jet Propulsion Laboratory & S. Mukherjee & Jawaharlal Nehru University \\
\hline V. Chevrier & University of Arkansas & M. E. Banks & NASA GSFC \\
\hline T. Meng & Lunar and Planetary Laboratory & P. A. Johnson & University of Alberta \\
\hline B. Tober & Lunar and Planetary Laboratory & J. C. Johnson & University of Alberta \\
\hline S. Ulamsec & German Aerospace Center (DLR) & J. C. Echaurren & University of Santiago, Chile \\
\hline A. Khuller & Arizona State University & C. Dinwiddie & Southwest Research Institute \\
\hline S. Adeli & German Aerospace Center (DLR) & B. L. Henderson & n Jet Propulsion Laboratory \\
\hline L. R. Lozano & Université Catholique de Louvain & D. Lalich & Cornell University \\
\hline \multicolumn{2}{|c|}{ E. Rivera-Valentín Lunar and Planetary Institute } & S. Nerozzi & Lunar and Planetary Laboratory \\
\hline E. Petersen & University of Alaska Fairbanks & & \\
\hline F. Foss & Freestyle Analytical \& Quantitativ & ices, LLC & \\
\hline
\end{tabular}

\section{Signatories:}

$\begin{array}{ll}\text { R. Lorenz } & \text { JHU Applied Physics Laboratory } \\ \text { M. Day } & \text { University of California Los Angeles } \\ \text { M. Pajola } & \text { Astronomical Observatory of Padova } \\ \text { A. Lucchetti } & \text { Astronomical Observatory of Padova } \\ \text { C. Newman } & \text { Aeolis Research } \\ \text { L. Tamppari } & \text { Jet Propulsion Laboratory } \\ \text { M. Patel } & \text { The Open University } \\ \text { J. C. Stern } & \text { NASA GSFC }\end{array}$
J. Eigenbrode NASA GSFC
A. Brown Plancius Research
Ö. Karatekin Royal Observatory Belgium
C. Cesar Universität Bern
T. G. Cave Raytheon Technologies
M. Mischna Jet Propulsion Laboratory
P. Streeter The Open University
C. M. Dundas U. S. Geological Survey 


\section{Executive Summary: As the subject of a New Frontiers class mission in the next}

decade, Mars Polar Science could address numerous compelling Solar System-level knowledge gaps and human exploration needs.

1. The ice-rich martian polar layered deposits (PLDs) contain thousands of ice layers that record the climate history for over several million years, including several key orbital changes. The PLDs provide the most accessible and complete climate record outside the Earth, making them among the most compelling science targets in the Solar System.

2. The PLDs are a key part of a diverse and integrated climate and volatile deposit system spanning much of the planet, both above and below the surface. The Mars volatile and climatic system contains numerous active processes that interact with the near-surface, and the lower and middle atmosphere circulations, even influencing the upper atmosphere and atmospheric escape.

3. The martian poles are an archetype for numerous Solar System bodies and processes on other bodies including climate change dominated by orbital variations, glacial flow, vaporpressure supported atmospheres and sublimation-dominated landscapes.

4. Mars Polar Science is critical for future human exploration and in situ resources, technology development for other targets, as well as the study of planetary habitability.

Broadly construed, Mars Polar Science is intimately linked to understanding the whole planet's climate and volatile system and provides an important analog for other bodies in the Solar System. The integrated nature of the Mars climate system means that nearly every part of Mars is affected by what happens at the poles, both within the polar ice deposits and in the atmosphere, with activity occurring on seasonal, multi-annual, and orbital-change cycles. In this white paper, we lay out four compelling reasons to advance investigations of activity related to Mars Polar Science; each reason is an umbrella for a range of other rationales that are, in turn, worthy of further study.

Climatic processes relate to all major Mars Exploration Program Analysis Group (MEPAG) objectives: Life, Climate, Geology, and Human Exploration, making studying the climate of Mars among the highest priorities in the MEPAG goals document. Numerous polar-related factors contribute to the current climate, including 1) the present distribution and seasonal fluxes of $\mathrm{H}_{2} \mathrm{O}$ and $\mathrm{CO}_{2}$ ices, 2) their latitude-dependent long-term deposition or ablation at each latitude, 3) dust transport and atmospheric loading, and 4) the climate record stored in layered deposits. The present climate will eventually become part of the climate record. Because the physical processes acting today are similar to processes that acted in the recent past, and because these processes are integral parts of Life, Climate, Geology, and Human Exploration, learning about the present climate is of critical importance for all areas of investigations of Mars. Here, we present the case for advancing investigations of activity related to the polar atmosphere and ice deposits of Mars.

\section{Polar Layered Deposits are Among the Most Compelling Solar System Science Targets}

Unlocking the formation history and climate record in the polar layered deposits (PLDs) would be a seminal advancement in NASA's goal to understand the climate systems of terrestrial planets. The PLDs are predominantly icy, $1000 \mathrm{~km}$ across and a few kilometers thick (Figure 1). Their size, composition, and layered nature are similar to Earth's Greenland ice sheet (which has long been a target of paleoclimatic study). Since their discovery, scientists have hypothesized that the PLDs would contain a detailed climatological record (1), like on Earth, because early modeling work showed that orbital forcing should affect the stability of ice at the poles (2). 
After nearly five decades of observations and modeling, scientific consensus has converged on a leading hypothesis in which the detailed climate record of the North PLD (NPLD) spans $\sim 4$ Myr (3). In this hypothesis, the NPLD accumulate at an average rate of $\sim 0.5 \mathrm{~mm}$ per year, but with some periods of rapid accumulation and others with widespread ablation. Ice lost in ablation periods recondenses in the mid-latitudes in an "ice age" cycle that closely tracks variations in insolation as a function of changing planetary obliquity, eccentricity, and argument of perihelion (3-7). Even within this consensus, knowledge gaps remain, such as whether the current NPLD are gaining or losing mass. Confidently answering these questions is not possible with current instrumentation.

Our highest resolution orbital cameras observe layers down to $\sim 1 \mathrm{~m}$ on sloping surfaces, and radar observations reveal dozens of reflectors that correspond to variations in ice and dust content (Figure $1(8)$ ). Correlation between patterns in the 2000 meters of stratified NPLD and the evolution of Mars' obliquity suggest that observed layers reveal a temporal resolution down to $\sim 2$ kyr. Our observations guarantee that thousands of data points are waiting to test our current hypothesis, a sampling rate of $\sim 100$ per full $\sim 100 \mathrm{kyr}$ obliquity period, and the full resolution of climate history may be much greater. An in situ mission to sample layers should provide several critical measurements [WPs Becerra et al.; I. B. Smith et al.; ICE-SAG 2019] including at least:

- Paleoatmospheric composition at each layer from trapped gases

- Both the crystalline state and bulk density of the ice as a function of depth

- Deuterium to hydrogen $(\mathrm{D} / \mathrm{H})$ ratios from the vertical column of the PLD

- Stratification of non-volatile material compositions and volumetric ratio to $\mathrm{H}_{2} \mathrm{O}$; this includes atmospherically derived dust, salts, and lithic materials, including possible ash or lithic layers from volcanic or impact events and possible alteration minerals that formed in place

From those measurements, we can derive critical, new information regarding:

- Absolute ages from lithic materials to determine the dates of climatic events

- Orbital forcing timescales, testing the widely accepted hypothesis about recent history (9)

- Dust accumulation rates and content through time, tying to the planetary dust cycle

- Lithic composition through time, tying to past atmospheric redox conditions

- Mars atmospheric loss rates, especially hydrogen

Addressing these critical knowledge gaps would fill a hole in our understanding of Mars history.

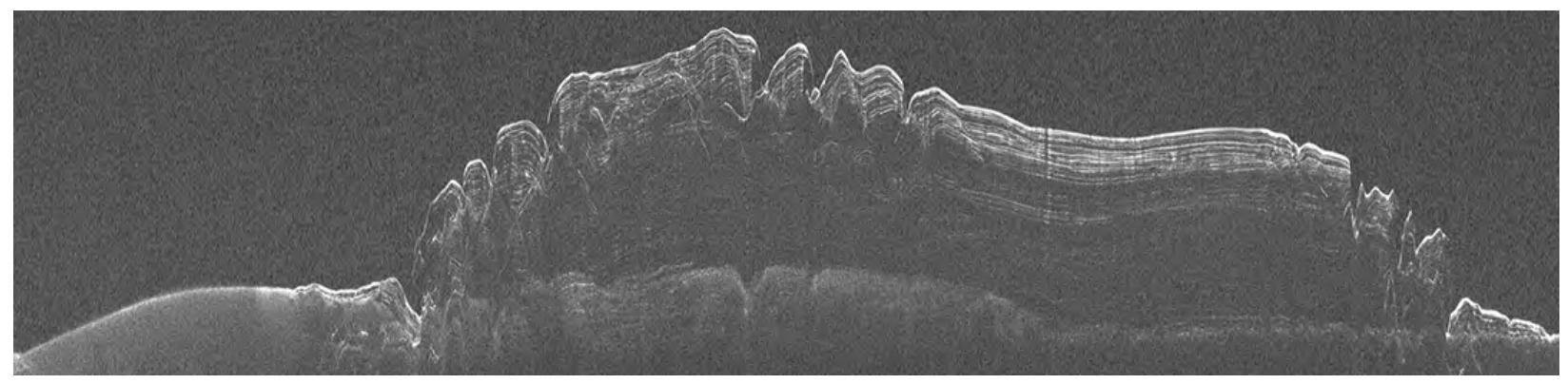

Figure 1: Shallow Radar (SHARAD(10)) observation showing dozens of layers and stratigraphic properties of a sedimentary deposit that contains a detailed climate record.

\section{Why investigate the NPLD?}

The maturity of Mars Polar Science, demonstrated by the hundreds of recent papers on ice, atmospheric and seasonal processes, and climate in the last ten years, makes the NPLD an extremely compelling target in the Solar System. Our recent work as a community has produced 
detailed hypotheses about the formation of the NPLD in the recent obliquity regime $(3,4)$ that is backed by modeling, laboratory experiments, and abundant data analysis. Missions conceived for polar exploration (ICE-SAG 2019; WP Smith et al.; (11)) would test those hypotheses directly and answer questions that have been pondered for decades.

Climate scientists have learned a great deal about our past and present climates on Earth by studying ice cores from the Arctic and Antarctica. Thus, precise determination of the 1) age; 2) stratigraphy, including layer thickness; 3) layer composition for isotopologues (especially D/H), salts, and lithic material (especially dust fraction); and 4) unconformities representing climatic shifts, is critical to constraining past climatic states and processes. The Mars Polar Lander mission was designed to fill critical knowledge gaps when selected over 20 years ago. The compelling nature of such a mission has only increased since then. Simply put, we know that fascinating, groundbreaking, conclusive science is uniquely accessible and ready to be investigated; deciphering the layers would be one of the greatest triumphs in planetary science.

\section{Mars Polar Science as a Diverse and Integrated System:}

Examples of polar investigations that have been submitted to this call for white papers include the climate record stored in the polar layered deposits [WPs Becerra et al.; Smith et al.,]; the midlatitude ices processes and climate record [WP Bramson et al.]; active surface processes [WP Dundas et al.]; glacial and periglacial processes [WP Grau Galofre et al.]; wind and frost mechanisms [WP Diniega et al.]; dust transport [WP Newman et al.]; and atmospheric circulation [WP Guzewich et al.]. These study areas are intimately linked such that learning about one subsystem informs all the others.

A large body of work has been completed within the last five years to define science priorities (MEPAG Goals Document, 2020) and missions to address these priorities. Three reports in particular broadly address Mars Polar Science: ICE-SAG (ICE-SAG 2019; WP Diniega et al.), NEX-SAG (WP: Zurek and Campbell: Next Science Analysis Group), and KISS (WP: Smith et al.: Keck Institute of Space Studies (11)). In this section of our white paper, we reiterate several of the most important areas for future study to advance the entire field.

\section{Ice Deposits:}

Recent and ongoing missions have found and continue to find new solid ice reservoirs on Mars, for both water and carbon dioxide ices, and there are many opportunities to study them.

Carbon dioxide ice is present over various locations and on various timescales, from ephemeral clouds, snowfall (12), diurnal frost, and seasonal caps (13-15), to the south pole residual cap (SPRC) $(16,17)$. The thin, high albedo SPRC that persists on decadal to century time scales was of primary interest for early Mars observations (18). This unit overlies part of the roughly domeshaped South PLD (SPLD) but sits offset from the geographic pole and covers the highest elevations (19-21). The SPRC, with an estimated mass that is $\sim 1 \%$ that of the full atmosphere, records the gains and losses of $\mathrm{CO}_{2}$ over the last 100-200 Mars years (22). Since the writing of the previous decadal survey, massive deposits of $\mathrm{CO}_{2}$, exceeding the mass of the martian atmosphere, were discovered beneath the SPRC (23-25). Gaps in our knowledge of their inherent properties, climatic implications, and relationship to the SPRC motivate further investigation, especially about the thickness, distribution, and internal stratigraphy (24).

We have known for decades about reservoirs of water ice that include the PLDs, including the basal units of the NPLD called Rupes Tenuis and Cavi (26). Lobate debris aprons were identified during the Viking missions, and recent and ongoing studies of the Eastern Hellas region $(27,28)$, Deuteronilus Mensae (29), Phlegra Montes (30), and Argyre Planitia (31) have confirmed the 
presence of water ice and have estimated their volume $(32,33)$. Significantly, since publication of the previous decadal survey (34), several mid-latitude units comprising large volumes of ice have been discovered at Arcadia Planitia (35), Utopia Planitia (36), and in the southern mid-latitudes (37), in addition to the glacier-like landforms in locations listed above. The Subsurface Water Ice Mapping (SWIM, (38)) project is underway to map ice deposits between $-60^{\circ}$ and $60^{\circ}$ latitude. These ice deposits contribute large volumes to the planetary water budget and offer resource targets for future human exploration. However, the spatial distribution and total budget is not well constrained, as is the effect on active or past weathering processes (39). Further, higher resolution ice concentration mapping and stratigraphy within the upper meter or so (the region that would exchange on orbital time scales) is necessary. The search for ice continues, especially at depths of 1 and $15 \mathrm{~m}$, where current instruments are blind except for recent impacts that expose ice $(40,41)$. Mission concepts such as Mars Ice Mapper [MEPAG \#38, 2020], MORIE [WP Calvin et al.], and MOSAIC [WP Lillis et al.] seek this ice.

These mid-latitude ice deposits are critical data points for climate history, both because of the geologic processes that act presently and have acted on them in the past, and because of their link to the polar record. They probably respond to the same forcings as the PLDs (e.g., transfer of volatiles and dust from one to the other) but are frequently out of phase. However; there is growing evidence that the mid-latitude ice can persist over timescales much longer than known orbital variations (42). Missions that would study the mass balance of water ice and vapor at the midlatitudes to determine accumulation and ablation rates, would fill a knowledge gap about the longterm climatic implications [WP Bramson et al.; WP Diniega et al.: ICE-SAG]. Balancing that with in situ polar mass balance measurements would definitively answer questions about climate processes and the transfer of ice between latitudes.

\section{Surface Activity:}

The ice deposits discussed above interact with the atmosphere, generating changes to atmospheric composition (43) that may determine the rates and locations of numerous surfaceatmosphere interaction processes. Of particular importance are the annual processes that drive long term migration of ice between latitudes, including sublimation, lag formation, and atmospheric circulation that transports volatile fluxes (11). Many locations experience diurnal and seasonal surface frost that causes geomorphological activity [WPs: Diniega et al.; Dundas et al.] and interacts with dust and water ice on the surface. Monitoring evolution of the seasonal frost and interactions between dust and water ice is of critical importance.

Numerous active processes have been interpreted at the PLD, especially related to the migrating spiral troughs and winds that drive them. Katabatic winds in spring and early summer drive landscape evolution (44-47). These winds form katabatic jumps, an intrinsically interesting phenomenon that also exists on Earth, which are hypothesized to give the spiral troughs their characteristic shape and wavelength. Thus, katabatic winds, along with large eddy circulations (e.g., (48)), force topographic changes that can be read from the ice record. Surface morphology might be used as a proxy for identifying accumulation or ablation areas. Whereas the redistribution of ice by wind at the surface of the polar caps is observed and is a critical agent for evaluating the surface mass balance, ice transport mechanisms and respective fluxes remain poorly characterized. At the margins of the NPLD, some scarps are currently the most active known places on Mars with annual avalanches and block falls during spring. Measuring the volumetric loss of material causing scarp retreat, and comparing rates to expected outward motion from ice deformation, constrains models of NPLD rheology and evolution (49). Detecting and measuring sublimation rates of both subsurface and surface seasonal frost using in situ instrumentation, along with concurrent 
measurements of meteorological drivers, would support much broader studies of climatic importance. Additionally, continued monitoring of surface activity from orbit would provide the broader context of frost's influence on surface changes.

\section{Atmosphere and Climate:}

Mars Polar Science is intrinsically tied to atmospheric and climate science as all transport of volatiles routes through the atmosphere. Knowledge gaps related to the atmosphere are numerous, especially at the poles, and investigations from orbit and in situ would help close those gaps and bolster our understanding of the integrated system [(11); WPs: Bapst et al.; Smith et al.; (11)]. Investigations that measure fluxes, sources and sinks of water, and quantify the present-day water cycle are critical to understanding the mass balance of ice at the surface. Investigations that determine how the polar vortices (1) partition the atmosphere, (2) concentrate non-condensable gasses, (3) distribute water ice clouds, (4) track latent heat released from $\mathrm{CO}_{2}$ condensation, and (5) activate polar dust storms will inform all parts of polar science. Seasonal condensation of $\mathrm{CO}_{2}$ affects the gravitational signature and moment of inertia of Mars (50) by loading the surface, affecting length of day (51). By measuring the present atmospheric transport along with polar ice mass balance, we can understand the processes that act today and extend that understanding into the past. To address this, we need to ask and answer the following:

- What are the global volatile and dust budgets?

- What is the distribution of water vapor and clouds above the polar caps through a full year?

- What are the current fluxes of volatiles and dust into polar regions from lower latitudes?

- How are volatile and dust fluxes affected by global and regional dust storms?

- How is water exchanged and transported between and through atmospheric, surface, and subsurface reservoirs?

- Is the water cycle now in an approximately steady state or in transition between climate states, and, if the latter, what is the rate of that transition?

- What is the current $\mathrm{D} / \mathrm{H}$ ratio at the surface and in the air above the PLD through a Mars year?

- What processes occurring at the surface of the ice deposits today will be recorded as layers?

Refining and developing high-resolution global climate models (GCMs) and mesoscale models over the PLDs $(48,52,53)$ is a necessary step in understanding the connection between the polar climate record and the climates which formed them. Significant advances in simulation capabilities have been made in recent years, especially since the Vision and Voyages document was published. However, modelers require more measurements for validation and assimilation to fully characterize the climate system. Of special importance are the poorly known global wind fields and vertical structure of atmospheric water vapor [WPs Guzewich et al.; Newman et al.]. Once validated in the present day, these models can be adjusted to look at past orbital/axial states helping us to understand how Mars became the planet it is today.

The upcoming Emirates Mars Mission (54), along with the Trace Gas Orbiter (TGO), Mars Reconnaissance Orbiter (MRO), and Mars Atmosphere and Volatile Evolution mission (MAVEN) missions all contribute to this knowledge, but significant gaps remain, including critical activity in the lowermost scale height, where all surface-atmosphere interactions occur. Polar and atmospheric research of Mars in the coming decades can get a significant boost through similar orbital and landed missions that focus on the lower atmosphere and investigate the PLDs [WPs Kleinboehl et al.; Smith et al; (11)]. 


\section{Mars as an Analogue to Solar System Bodies}

Ices are found on many bodies in the solar system, from Mercury to the Trans-Neptunian bodies Pluto and 486958 Arrokoth. On Earth, we experience a temperature and pressure regime that only permits one volatile to readily change phase from gas and liquid to solid and back; however, bodies farther out in the Solar System exhibit this process for numerous other volatiles such as $\mathrm{CO}_{2}$, molecular nitrogen, carbon monoxide, methane, and more. Most of these volatiles are found only in the solid or gaseous phases. On Mars, two volatiles exist in the solid and gaseous phases, providing context and analogous behavior for bodies farther out from the sun. In particular, $\mathrm{CO}_{2}$ gas is the primary constituent of the martian atmosphere and so acts as the primary climate driver, drives surface-atmosphere interactions, and acts as an agent of geomorphologic change in the solid and vapor state (13). Throughout Mars' recent history, going back to at least $\sim 1 \mathrm{Ga}$, the atmospheric pressure has oscillated from likely near-zero to approximately double the present value (55). In these oscillations, $\mathrm{CO}_{2}$ ice forms at the poles and mid-latitudes, affecting the surface, contributing to layered deposits, and possibly forming glaciers. At times, Mars even experienced partial atmospheric collapse (56), behavior analogous to Pluto/Triton (57-59) and even Titan (60).

Mars is the best-studied analogue to other planetary bodies [WP Diniega et al.]. Pluto (59) and Triton probably have layered ice deposits (perhaps of multiple volatile species), analogous to Mars' layered deposits. Further, both have dry cryosphere processes that exist on Mars but rarely on Earth. Some atmospheric examples include low-altitude $\mathrm{CO}_{2} / \mathrm{N}_{2}$ condensation for Hellas (Mars) and Sputnik (Pluto) Planitiae and sublimation/condensation-driven winds. Geologic examples include jets that form from the solid-state greenhouse effect (61-63), icy haloes on Mars and Triton (64), sublimation pits and terrain, thermally induced stress fracturing of icy deposits on Mars (65, 66 ) and within craters of icy moons like Europa, Ganymede, and Callisto $(67,68)$, and martian penitentes (69) that provide clues about those found on Pluto (70). Many more examples exist. Further, Pluto has been called a "frontier in glaciology" because it exhibits cold-based glaciation, similar to Mars and some locations on Earth. Umbriel, Triton, and probably Titan might have similar cold-based glaciation during favorable cycles. Additionally, Mars acts as an analogue for Earth in terms of modelling possible future climatic shifts (71). Having abundant water ice, the vast majority of which is locked into solid-phase reservoirs at the poles and low latitudes, makes Mars a great analogue for ice processes throughout the Solar System.

Lastly, Mars has ice deposits that form within the regolith that are more akin to homogenous mixtures than layered deposits. These deposits may act as analogues to inner solar system bodies such as the Moon, Mercury, Ceres, and even Earth. Investigating the processes that act on martian deposits with the missions that are supported in ICE-SAG and the KISS study (11) will improve our knowledge of the physics that drives volatile transport on other Solar System bodies.

Because Mars is a high-fidelity analog and more readily accessible than those bodies farther away, future missions to Mars should focus on understanding solid-gas volatile processes that likely play a role in changing the surface and the climate record for outer Solar System bodies. Thus, Mars Polar Science serves as a link between the classic Earth cryospheric sciences and exotic ice worlds, combining some characteristics of both, the "usual" and the "unusual," and making Mars a highly flexible natural laboratory for many Solar System targets.

4 Mars Polar Science is Critical for the Study of Astrobiology, Future Human Exploration and in situ Resources, and Tech Development for Other Planets 


\section{Astrobiology, Liquid Water, and Habitability:}

Detecting traces of extant life on Mars is a high scientific priority. Polar regions are an important target for assessing potentially habitable environments on modern Mars with permafrost, glaciers, and underlying regolith in PLDs being key sites in this search (72). Briny water may form ephemerally in the present martian climate (73-75), and liquid water is essential for life and habitability. The necessary conditions for deliquescence and possible melting to occur are more likely to be met in the martian polar regions than at low and equatorial latitudes (76), perhaps as thin films $(77,78)$, even to the point that evidence of a brine-like sub-SPLD lake has been presented (79). Brine formation is yet to be confirmed, but if it does, halophilic terrestrial bacteria can thrive in extreme, natural brines (80-82). The question of Mars habitability can never be fully answered without their study.

The low temperatures, anoxic conditions and lack of light in sub-ice environments promote unique biochemical pathways that sustain chemotrophic microbial communities on Earth (e.g. (83, 84)), making icy regions high value targets for astrobiology missions. Further, habitable conditions can occur in high-latitude ground ice up to $1 \mathrm{~m}$ deep at high obliquity as ground ice warms sufficiently to contain liquid water films (85). In the next decade, we can investigate, in situ, the kinetics of brine formation by deliquescence (80), the water budget in such brines, their contribution to the water cycle, and their planet-wide formation rate [WP Rivera-Valentín et al.]. Current technologies including nano-scale sensors, elemental composition analysis, and remote sensing devices have been engineered for enhanced classification of this evidence, however dedicated life-detection missions are rare. Astrobiological considerations further motivate investments in Mars Polar Science because the PLDs are the best place on the planet to seek potentially habitable brines and habitats because of long summer seasons with favorable energetic periods and the abundance of surface and near-surface ice.

\section{Human Exploration:}

Advancing Mars Polar Science benefits future human exploration in myriad ways. As exhibited by the MEPAG goals document, there is strong overlap between the high-priority science objectives outlined above and the science needed to prepare for human exploration:

- Shallow ice deposits as resources for human explorers

- Wind measurements for landing and habitat safety

- Dust composition, abundance, and mobility for human health

Human exploration goals provide additional motivation for exploring icy deposits on Mars.

\section{Collateral Advantages from Mars Cold Technology (Cold Tech) Development:}

Accessing the PLD with a lander and drill would provide collateral technology benefits. The technologies and methodologies for accessing the PLDs, including cryo-drills and other Cold Tech, is a necessary steppingstone for exploring icy moons in the outer solar system. Technology developed for Mars and deployed in this decade will benefit future exploration of those moons.

\section{Concluding Remarks:}

Numerous fields fit in the broader category of Mars Polar Science. Each component is compelling as a science target in the next decade. We recommend that the science listed above be a potential candidate in the decadal survey report for New Frontiers missions.

\section{REFERENCES: https://sharad.psi.edu/ ibsmith/files/White_paper_Mars_Polar_references.pdf}

